\title{
3D Interaction in Virtual Environments for Systems Engineering
}

\author{
M. Bougaa, S. Bornhofen, H. Kadima, and A. Rivière
}

\begin{abstract}
This paper discusses the potential benefits of applying Virtual Reality (VR) technology to the context of Systems Engineering (SE), for both educational and industrial purposes.

After an introductive presentation of the two fields and their state of the art, we explore if and how VR can be of assistance to the processes involved in a typical SE approach. We especially focus on commonly used 3D interaction techniques in VR and argue that the design of appropriate $3 \mathrm{D}$ interactions is a key ingredient for the success of VR in SE. We suggest three research directions that may be considered for this design: interaction generality, context awareness and adaptability. The 3D interactions should adapt, manually or automatically, to the VR device, the virtual scene and the user context.
\end{abstract}

Index Terms-Virtual reality, virtual environment, systems engineering, 3d interaction, adaptive interaction.

\section{INTRODUCTION}

Over the last decades, the engineering of physical systems and the management of their life cycle have grown more and more complex. Military, aerospace, automotive and medical industries are confronted with the challenge of building systems that have large architectures and require interdisciplinary competence. Moreover, the systems must not only be functional, but also reliable, maintainable and safe. As an example, Fig. 1 illustrates the multidisciplinary nature of the design process of an aerospace launch vehicle at the Onera research center [1].

The understanding and the design of such complex systems cannot be achieved any more by a simple Systems Design approach which is the concept, specification, implementation, verification and validation of a technical system for achieving a specific (and mostly functional) objective [2]. New goal-independent methodological approaches and modeling techniques are required, such as Systems Engineering (SE) whose first concern is not a specific design objective, but the optimization of all involved engineering processes. As defined by the International Council on Systems Engineering (INCOSE): "Systems engineering is an interdisciplinary

Manuscript received January 29, 2015; revised May 26, 2015. This work is done under PLACIS Project, driven by "l'Institut Polytechnique du Grand Paris" (SUPMECA, EISTI and ENSEA). PLACIS is funded by the french Ministry of Higher Education and Research under the future investments program with the reference ANR-11-IDFI-0029.

M. Bougaa, S. Bornhofen, and H. Kadima are with the L@RIS Laboratory - EISTI, Ecole Internationale des Sciences du Traitement de l'Information, Avenue du Parc, 95000 Cergy-Pontoise, France (tel.: +33 1-3425-1010; Fax : +33 1-3425-1000; e-mail: \{mba, sb, hk \}@eisti.eu).

A. Rivière is with the LISMMA Laboratory-SupMéca, Institut Supérieur de Mécanique de Paris 3 Rue Fernand Hainaut, 93400 Saint-Ouen, France (Fax :+33 1-4945-2900; e-mail: alain.riviere@ supmeca.fr). approach and means to enable the realization of successful systems. It focuses on defining customer needs and required functionality early in the development cycle, documenting requirements, and then proceeding with design synthesis and system validation while considering the complete problem: operations, cost and schedule, performance, training and support, test, manufacturing, and disposal. SE considers both the business and the technical needs of all customers with the goal of providing a quality product that meets the user needs". [3].

Much research effort has focused on proposing appropriate tools for SE. Dedicated modeling languages have been introduced, such as SysML [4], a visual modeling language, and LML (Lifecycle Modeling Language) [5], providing a simple way to understand and communicate cost, schedule and performance design information to all stakeholders in a standard manner. Other successful standardizations are ISO/IEC 15288 [6], defining a common framework for the life cycle of systems, EIA 632 [7] proposing the blueprint of all processes and requirements necessary for engineering a system, or IEEE 1220 [8] providing the next-level-of-detail description of SE processes defined in EIA 632. However, not all technologies that might support the SE approach had full attention of the industrial and academic communities so far, either because they are recent like Big Data, or because up until recently they were immature and unaffordable, like Virtual Reality (VR).

As a matter of fact, only little research has been done on VR for SE, although its potential has already been discovered especially for educational needs [9]. This paper pursues the assessment of the benefits of VR in the context of SE. We intent to develop a collaborative VR based environment for using and learning SE, we are particularly interested in the study of 3D interaction techniques and explore if and how they should be improved or adapted, in order for systems engineers to take maximum advantages from a VR environment.

The paper is organized as follows: Section II discusses the requirements to be met by a typical SE environment. Section III reviews the state of the art in VR, and distinguishes between the components sufficiently mature to be applied to $\mathrm{SE}$ and those that require improvements. Section IV focuses on immersive 3D interaction techniques and puts forward some directions to explore, in order to take VR to a higher level of maturity, in terms of precision and ease of use.

\section{SYSTEMS ENGINEERING ENVIRONMENTS}

This section identifies the requirements that need to be met by any appropriate SE environment in both academia and 
industry (not to be confused with the requirements of a system to be engineered). For this purpose, we consider the thirteen processes in a typical SE approach as defined by the EIA 632 standard [7]. The processes are illustrated in Fig. 2. They may be adapted to the specificities of the domain, the product type, and other industrial or pedagogical constraints.

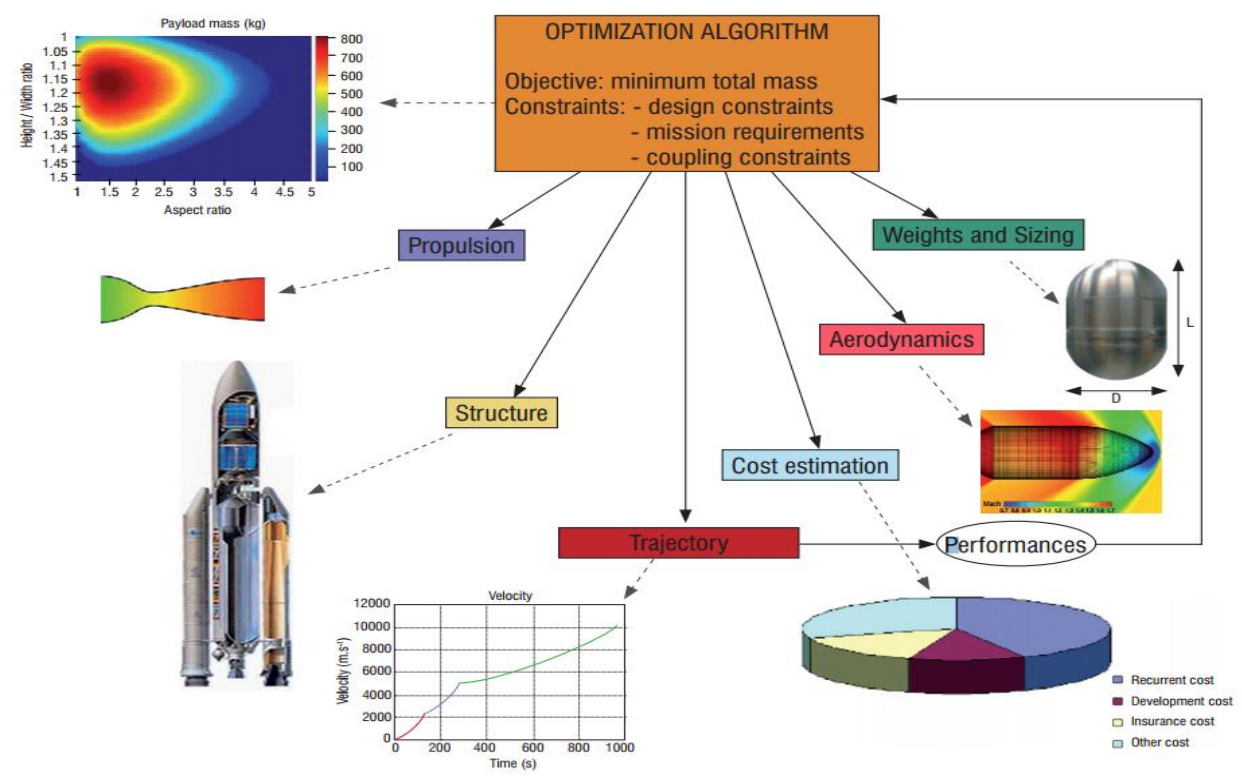

Fig. 1. Multidisciplinary design process of an aerospace launch vehicle [1].

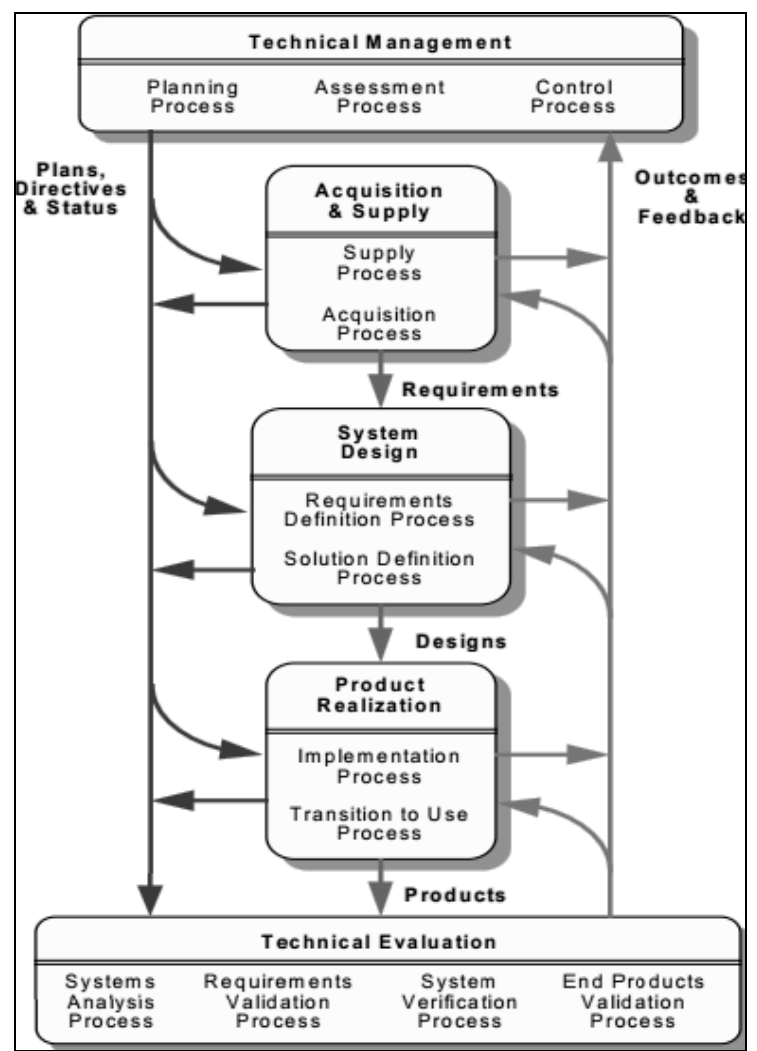

Fig. 2. Processes for systems engineering as defined by EIA 632 [7].

Abulrub et al. [9] explored the benefits of VR for engineering education and training through a series of case studies at the University of Warwick. The authors showed how VR can encourage the creative learning of engineering material and environments, and concluded that some VR promises for SE are: the development of autonomous problem solving skills, the sharing of complex information with team members, and the analysis of engineering problems under different points of view. Maurer and Winner [2] discussed the upcoming challenges for SE in the automotive and supplier industry. Some of them are: requirements engineering, the design of test and validation strategies and tool.

Based on these two similar works, we pre-selected five EIA 632 processes where the use of VR is particularly promising in the context of SE. These processes will be addressed in the scope of this paper (processes definitions taken from [7]):

1) Requirements Definition Process: transform stakeholder requirements into a set of system technical requirements.

2) Solution Definition Process: generate an acceptable design solution (specifications, drawings, models, etc.).

3) Implementation Process: transform the characterized design solution into an integrated end product that conforms to its specified requirements.

4) Requirements Validation Process: assure that the subject set of requirements describes the input requirements and objectives such that the resulting system products can satisfy the requirements and objectives.

5) System Verification Process: ascertain some points such as: the system design solution is consistent with the source requirements, the end products meet at each level their specified requirements, etc.

\section{A. Industry}

In order to enhance the SE approach in terms of efficiency, simplicity and flexibility, we particularly focus on the SE environment requirements to obtain better results during the five previously cited processes. In this section we illustrate the results of our reflection on the definition of these requirements, and we discuss which requirement affects which processes:

We identified some shared requirements for the two Systems Design processes, in order to improve the results of the Requirements Definition Process and the Solution Definition Process. The SE environment should offer tools and techniques to:

1) Improve the design of digital prototypes/mockups with 
higher quality and realism.

2) Enhance collaboration and break down distances and multidisciplinary barriers;

3) Help the engineers in their critical thinking and problem solving;

4) Adopt different points of view of the system, because the perception of the system depends on the engineer's scientific background;

5) Do efficient project reviews and increase the reliability of early-stage decisions;

6) Use knowledge bases in order to identify significant design issues at early stages.

Some of these requirements are shared between more than two processes, such as the points 5) and 6), which can highly affect costs and time-to-market and so they affect the Implementation Process. Point 4) has a significant impact on the Requirements Validation and the System Verification Processes.

In addition to these system design requirements, we identified one requirement for the Implementation Process, which is the ability of the SE environment to offer tools for:

7) Efficient task and time planning as well as resource management to improve the implementation process,

Two other requirements for the Requirements Validation and the System Verification Processes:

8) The ability to ensure the system compliance with the requirements;

9) Efficient tools for knowledge sharing, transmission and validation,

And finally, one shared requirement for the last three processes:

10) The use of techniques such as Failure Mode and Effects Analysis (FMEA) techniques [10] in virtual prototyping, for realistic simulations, in order to prevent systems assembly or behavior problems and defaults at early stage of the engineering process.

Fig. 3 summarizes these requirements and specifies which process is concerned by each requirement.

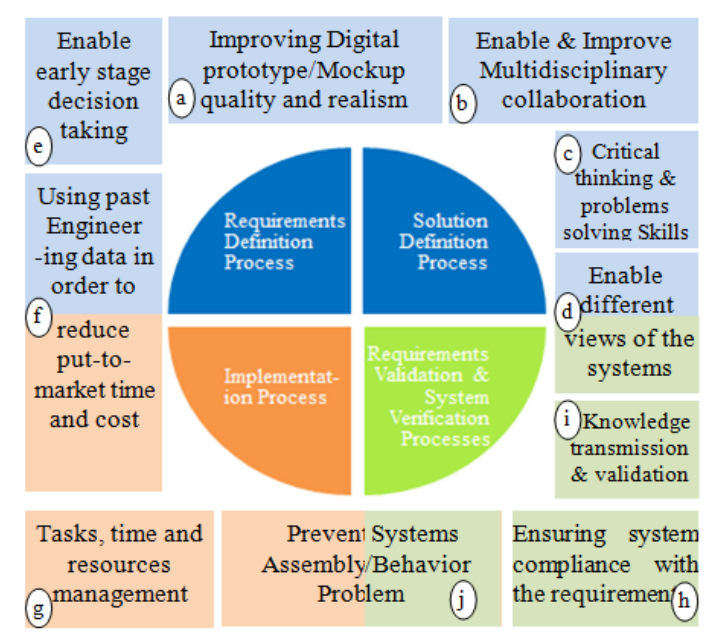

Fig. 3. Processes (quadrants) and their requirements (rectangles).

\section{B. Academia}

A good SE platform should provide the students with tools to acquire essential skills [9], [11]. Naturally, many of the requirements for SE education and learning solutions are identical to the previously cited requirements for SE in industry, such as getting the user to learn how to:

1) Ensure system performance and functionality by respecting compliance with the requirements during the engineering processes;

2) Optimize task, time and resource management;

3) Adopt the best practices for collaborative work, especially in geographically dispersed, multidisciplinary teams.

4) Acquire critical thinking and problems solving skills for real life engineering challenges;

5) Understand the benefits of inspecting engineering problems from different points of view (global view, components view, functional view, conceptual view, etc.);

6) Be able to make key decisions since the first stages of the engineering processes, especially encouraging innovation;

On the other hand, SE learning environments also have some specific requirements like:

7) Giving the ability to the instructor to fix learning goals before the training phase, in order to assess and validate the students' results.

8) Enable engineering students to experience an environment or a problem when it is difficult to do otherwise in real-life [9]

9) Encourage active participation and amplify student interaction and collaboration [9].

\section{VIRTUAL REALITY FOR SYSTEMS ENGINEERING}

After having reviewed the requirements for the engineering processes that we are focusing on, this section inspects which VR components are already mature, and suggests how they could be integrated in a SE environment.

VR is a technology that allows users to immerse in an artificial reality and to have interactive experiences via sensorimotor channels [12], [13]. The first VR systems appeared more than 50 years ago, such as the Sensorama simulator which it is one of the earliest known examples of immersive, multi-sensory (multimodal) technology [14]. However, VR has been democratized only in the last decade, and therefore has in many ways the flavor of a new research field. It is experiencing a rapid growth, as well as various related technologies: gesture control, speech recognition, gamification, wearable user interfaces, brain computer interface, etc.

The quality of the user experience in a VR system can be evaluated through several components. J. Tisseau considers that a VR application is defined as a function of three components [15]:

1) Autonomy: A behavior will be considered as autonomous, it if can adapt to unknown changes in its environment: meaning it must have the means to perceive, act and coordinate perceptions and actions, to be able to react realistically to these changes [16];

2) Immersion: exposing the user to a virtual environment using fully or partially real-world occulting devices [17];

3) Interaction: a communication language between the user and the machine. It is a set of actions/reactions via 
sensorimotor interfaces and interaction techniques [18]. Burdea and Coiffet defined the basic VR components as Immersion, Interaction, and Imagination [19]: Imagination characterizes the interpretation of the parameters that result from a VR experience.

If we want to apply VR in the context of SE, these components must be taken into account, especially the Immersion and Interaction components.

\section{A. Industry}

\section{1) The immersion dimension}

Immersion is a key element for efficient VR solutions, though its quality depends on the equipment and the techniques used for visualization and interaction. First, good immersion allows for a better perception of the environment. As a matter of fact, it has been shown that users estimate the depth of 3D models with greater accuracy using stereoscopic immersive systems compared to traditional display systems [20]. Second, immersion enhances the sense of presence especially by real-time user tracking and by visual feedback of the user's hands and body [21], [22]. Third, it enriches the possibilities of $3 \mathrm{D}$ interaction techniques for grabbing and manipulating virtual objects [23].

We claim that the immersion component of VR could considerably improve the SE processes requirements. The quality and the realism of digital prototype/mockup using VR allow for more reliable decision making, and therefore reduce costs and time-to-market. Another improvement resides in the use of failure analysis techniques, such as FMEA/FTA [10], and knowledge bases coupled to the 3D content, describing the object attributes and their interaction at early stages of the design. This approach helps preventing system anomalies that may occur in the later realization stage [24]. As a conclusion, the immersion component of VR can allow for a comprehensible 3D visualization of SE data and therefore improve the Requirements Validation, System Verification and Implementation Processes.

\section{2) The interaction dimension}

We can differentiate between two types of interaction in a VR based SE environment: the interaction with other users which are collaborating to engineer a system and the interaction with the virtual environment itself. VR and recent technologies offers mature tools for the first category, such as the collaborative design in virtual environments [24], improving collaboration and diminishing distance and multidisciplinary barriers. For the second category, the situation is more difficult. There is a variety of well-known 3D interaction techniques in VR, addressing all human senses including haptics and force feedback [23], [25]-[27]. However, their precision, accuracy, availability, and adaptability do not always deliver a good performance [28], [29]. For example, interaction with objects in a crowded virtual environment is different to a non-crowded one, especially for SE where the user typically does not interact with the entire complex system, but only with a part of it. Similar problems arise for tasks that need high precision interaction, compared to other tasks that do not need such accuracy. Many excellent states of the art on 3D interaction have been done in the past [26], [28], [30], [31]. Improving and adapting these techniques in the context of SE is the main subject of our work. We will study this topic in section 4 and suggest some promising research avenues.

\section{B. Academia}

Thanks to the interaction and immersion components that provide the students with a high level of realism and interactivity, VR is a well-suited tool for education and training. It offers a safe, fully controllable and cost-effective learning experience. VR teaches the students how to develop autonomous problem solving skills, and gives the instructor the ability to create realistic learning situations which are difficult, unaffordable or even impossible to set up in a classic learning context.

It has been shown that teaching and training is considerably improved by having the students apply theoretical knowledge to concrete industrial problems using VR technologies. Creativity, innovation, communication, problem solving, team work and business skills can be improved by using VR environments, which offers an unlimited experience on virtualized real-life situations [9].

\section{3D INTERACTION AND POSSIBLE IMPROVEMENTS}

This section discusses the current state of the art on 3D interaction techniques. We present the most famous ones together with some possible classifications and review a number of improvements that have been done until now. We conclude by suggesting some promising leads on what may be the future of $3 \mathrm{D}$ interaction techniques.

\section{A. Interaction Techniques and Classifications}

3D interactions have often been classified into distinct virtual behavioral primitives [13], [32]:

1) Techniques for observing the virtual world;

2) Techniques for moving and acting in the virtual word ;

3) Techniques for communicating with others or with the environment.

Another approach consists in classifying the techniques according to two main categories:

1) The egocentric techniques, where the user interacts with the environment "from the inside", as in reality, using his hands to select a virtual object;

2) The exocentric techniques where the user interacts with the environment "from the outside", meaning that the user is not considered as a part of the virtual scene;

Each of which has three sub-categories: direct manipulation, physical control and virtual control techniques [31].

Most often, a 3D interaction technique is related to an interaction task, which also allows classifying interaction techniques according to elementary tasks: navigation, selection, manipulation, and application control [33].

As two of the most frequently used 3D interaction techniques, we cite

1) The virtual hand metaphor [34], where the user touches the virtual object with his hand to select it, then he validates the selection by staying in contact with the object for a certain lapse of time or by closing his hand.

2) The ray-casting technique [35] which is a pointing 
technique based on the virtual ray metaphor, where an infinite laser pointer extends from the virtual hand of the user and traverses the virtual environment. The first object that intersects the ray can be selected.

\section{B. Limits of $3 D$ Interaction Techniques}

The two previously cited techniques have several drawbacks. Ray-casting suffers from the fact that the object is attached to the end of the ray, so there is no simple method to apply rotation to the selected object. The virtual hand technique suffers from the difficulty of grabbing remote objects.

For these reasons, a variety of new techniques have been explored. The improved virtual pointer metaphor [36] and the flexible pointer [37] enhance the ray-casting selection by allowing the control of the ray direction and therefore the selection of hidden objects. Also, several arm-extension techniques like the go-go technique [38] and its variant fast go-go [39] are improvements over the classic virtual hand for grabbing and manipulating remote objects. The go-go technique [26] gives the user the ability to select and manipulate distant objects, by calculating the virtual hand position using a non-linear function allowing it to go further than the real user hand after a certain distance threshold. The fast go-go technique is similar to the go-go technique, except that it does not have a distance threshold.

Such improvements may work in a specific virtual environment, however in a complex environment for SE, they can quickly become unusable. We illustrate this argumentation by a concrete use case. One of the interaction improvements is an adaptive technique where the user performs the navigation, selection and manipulation tasks sequentially, and the system switches automatically from one task to the next [40]. In some specific SE scenarios this technique may be very helpful and can save the user much time and cognitive load: in the case of a virtual system assembly with hundreds of pieces, the user repeats for each piece the same series of operations (navigate to the piece, select it, and put it in the right place). However, for another engineering problem where the user manipulates the products in a virtual production line, he does not need to use the navigation task neither the selection task because the objects can be automatically picked by the system. In this case, the rigid implementation of the previous technique would not be helpful.

This example illustrates we need some kind of adaptation possibilities for interaction techniques, we will discuss about adaptation at the end of the next section.

\section{New Directions}

As we have seen, 3D interaction is a key component of VR, and more research effort should be invested in order to conceive new interaction techniques or improve on the existing ones. In this section we suggest some directions where this research might concentrate on. We start by assuming some requirements which the 3D interaction techniques may need to satisfy in the same virtual environment:

1) For two different functionalities, we may use two different selection techniques, with the same or different devices, or even by natural interaction;

2) To achieve a navigation or manipulation task, more than one technique may be used simultaneously;

3) The interaction technique may change between two tasks.

\section{1) Generality vs. specificity}

Encouraging specificity when designing 3D interaction techniques is the first recommendation among four proposed in [41]:

1) Encourage specificity: design specific $3 \mathrm{D}$ interactions for specific domains, specific tasks, specific devices, and specific users.

2) Produce variants of existing 3D interaction techniques: by adding features or complexity to the basics techniques, to improve their usability.

3) Address the implementation issues: improve the implementation of 3D interactions by allowing the 3D interactions to handle a greater amount and variety of input data, create standards for 3D interactions, etc.

4) Apply 3D interactions to emerging technologies or technological concepts, such as wide-area tracking technologies, pervasive computing technology, etc., which also provides an opportunity for further 3D interaction research.

Although there is no guarantee that any interaction technique produced by this method (specificity) can be reused in any other domain, the authors suggest designing dedicated interaction techniques for each domain or application from scratch. Based on a specificity approach and not a generality approach, the authors recommend designing techniques for specific tasks rather than for a general task category. They also suggest device-specific techniques, and user-specific techniques.

We do not completely agree with this proposition. We think that focusing on specificity, especially application specificity will increase the number of 3D interaction techniques, and as a consequence future developers will be lost in choosing from existing techniques. Moreover, developing a new technique for each application and for each device is definitely not the best solution in order to make VR more usable.

We assume two conditions for this purpose: first, the interaction technique must be intuitive and ensure minimum cognitive load, by encouraging task continuity [40]. Second, after analyzing and decomposing all existing techniques into low level primitives, we propose that all of them should be grouped into one smart interaction solution. Keeping that in mind and that domain specificity may be encouraged, but not application specificity, our first research direction is:

\section{("Find a compromise between specificity and generality, and forget about the classic task, user behavior, or device type classifications of VR interaction techniques"}

\section{2) Context-awareness and user intention}

Previously, we suggested considering generality rather than specificity, but this approach does not have to prevent the system from taking useful environment information into consideration when performing interaction tasks, such as:

1) The extent of the virtual environment and information 
about the objects inside it: shapes, object sizes and density, inter-object relations...etc.

2) The task scenario: what does the user need to do next?

3) Detecting user intention based on previous uses of the system. Hence, our second research direction is:

"The interaction solution must be smart and have the ability to react according to the environment information as well as to the user behavior, to be more suitable and more accurate to the task requirements."

\section{3) Adaptative interaction techniques}

Keeping in mind the two previously proposed directions, we can note that the interaction technique solution to be designed needs to be adaptable, according to the retrieved information from the virtual environment, task requirements, and user intention. Some initiatives have already been undertaken to adapt basic interaction techniques, such as:

1) Switch between two or more interaction techniques when manipulating virtual objects in the same virtual environment.

2) Adapt an existing technique itself, for specific context use.

3) Improve existing techniques by including new interaction modalities, like speech control, eye tracking, haptic devices, etc.

4) Automatic execution of a part of the interaction task, like automatic selection of a specific form/color object, etc.

Many benefits of such adaptations have been highlighted, including: acceleration, personalization and diminution of cognitive load [42]. Even though our vision of adaptive 3D interactions implies the implementation of already conceived basic adaptations, we do not suggest cumulating individual adaptations. We rather suggest the implementation of these adaptations in the scope of one single interaction framework, according to our two previous research directions. Our goal is not providing assistance to the existing interaction techniques, but creating a fully adaptive 3D interaction solution. Therefore, our third direction is:

"After breaking down all the existing interaction techniques into low level primitives and the proposition of other required ones for each specific domain, we suggest the conception of one single adaptive solution regrouping all the interaction primitives."

Some kinds of adaptation that need to be taken into consideration for the creation of an adaptive $3 \mathrm{D}$ interaction technique in virtual environments are:

1) Adaptation performed automatically according to the interaction requirements, or manually by the user.

2) Adaptation according to the task requirement, to the user intention and to the task scenario analysis.

3) Adaptation according to the environment: the size of the object to be selected, object-object and user-object distances, velocities...etc.

4) Multi-device and multimodal adaptations: for a multi-user VR environment, featuring different devices, different modalities, etc.

5) Adaptation based on the user purpose: for a student, acquiring skills is more important than simplified interaction. In contrast, to systems engineering experts, the interaction must be as intuitive and as accurate as possible.

Each adaptation may be part of the previously cited initiatives (switching between two interaction techniques, the adaptation of the existing technique itself, improvements using new modalities, the automatic execution of part of the task), or they may be of another kind, such as adapting the user point of view (zooming or redirection), the geometry of the $3 \mathrm{D}$ cursor, etc.

\section{CONCLUSION AND FUTURE WORK}

In the field of VR, much work has addressed the design of 3D interaction techniques in virtual environments, but the results are not sufficient to democratize the use of VR technologies in certain domains, such as Systems Engineering. This paper suggested some new research directions to focus on, in order to improve future VR systems for industrial and educational needs, by improving the accuracy and the adaptability of 3D interaction techniques.

We plan on continuing our research and consider this paper as a starting point for our next contributions. The implementation of a VR environment for systems engineering in the automotive industry is already in progress.

\section{REFERENCES}

[1] S. Defoort, M. Balesdent, P. Klotz, P. Schmollgruber, J. Morio, J. Hermetz, C. Blondeau, G. Carrier, and N. Bérend, "Multidisciplinary aerospace system design: principles, issues and onera experience," Onera, AerospaceLab Journal, no. 4, p. 11, May 2012.

[2] M. Maurer and H. Winner, Automotive Systems Engineering, Springer, Heidelberg, 2013, ch. 1, pp. 3-35.

[3] Systems Engineering Handbook, a Guide for System Life Cycle processes and Activities, v. 3.2.2, INCOSE, October 2011, ch. 1, pp. 7-8.

[4] L. Balmelli, "An overview of the systems modeling language for products and systems development," Journal of Object Technology (JOT), vol. 6, no. 6, pp. 149-177, July 2007.

[5] Lifecycle Modeling Language (LML) specification, v 1.0, Technical Report. (October 2013). [Online]. Available: http://www.lifecyclemodeling.org/spec/LML_Specification_1_0.pdf

[6] Systems and Software Engineering -System Life Cycle Processes-, ISO/IEC International Standard, ISO/IEC 15288, $2^{\text {nd }}$ ed., 2008.

[7] Processes for Engineering a System, ANSI/EIA Standard, EIA-632-1998, Approved: January 1999.

[8] Standard for Application and Management of the Systems Engineering Process, IEEE Standard, IEEE 1220-1998, 1998.

[9] A. G. Abulrub, A. N. Attridge, and M. A. Williams, "Virtual reality in engineering education- the future of creative learning," presented at IEEE Global Engineering Education Conference, Amman, Jordan, April 4-6, 2011.

[10] S. N. Gaikwad and M. M. Mulkutkar, "Reliability based design with FMEA and FTA," IOSR Journal of Mechanical and Civil Engineering (IOSR-JMCE), vol. 5, pp. 21-25, 2013.

[11] M. Mihelj, D. Novak, and S. Bagus, "Interaction with a virtual environment," Virtual Reality Technology and Applications, Springer Netherlands, 2014, pp. 205-212.

[12] A. Berthoz and J. L. Vercher, The Treaty of Virtual Reality: Foundations and Behavioral Interface, $2^{\text {nd }}$ ed., vol. 1, Ecole des Mines press, France, 2006, ch. 1, pp. 3-30.

[13] P. Fuchs, G. Moreau, and P. Guitton, Virtual Reality: Concepts and Technologies, $1^{\text {st }}$ ed., CRC Press, Fla, USA, 2011.

[14] M. L. Heilig, "Sensorama simulator," U.S. Patent 3050 870, August 28,1962 .

[15] J. Tisseau, "Virtual reality and complexity," Scientific Manifesto, Ecole nationale d'ingénieurs de Brest, France, 2004. 
[16] J. Tisseau, "Virtual Reality: autonomy in virtuo," HDR dissertation (authorization to direct researches), Rennes I university, France, 2001.

[17] J. M. Burkhardt, B. Bardy, and D. Lourdeaux, "Immersion, realism and presence in the conception and the evaluation of virtual environments," French Psychology Journal, vol. 48, no. 2, pp. 35-42, 2003.

[18] L. Sternberger, "Interaction in virtual reality," $\mathrm{PhD}$ thesis, Louis Pasteur University of Strasbourg 1, France, 2006.

[19] G. Burdea and P. Coiffet, Virtual Reality, Hermès-Paris, ch. 2, pp. 243-251, 1993.

[20] F. Girbacia, A. Beraru, D. Talaba, and G. Mogan, "Visual depth perception of 3D CAD models in desktop and immersive virtual environments," International Journal of Communication, vol. 7, no. 5, pp. 840-848, December 2012.

[21] G. Bruder, F. Steinicke, K Rothaus, and K. Hinrichs, "Enhancing presence in head-mounted display environments by visual body feedback using head-mounted cameras," in Proc. International Conference on CyberWorlds, IEEE Press, 2009, pp. 43-50.

[22] S. Satyavolu and P. Willemsen. Enhancing user immersion and natural interaction in HMD based virtual environments with real time visual body feedback using multiple Microsoft Kinects. [Online]. Available: http://www.d.umn.edu/ saty0010/Midgraph2011_Satyavolu_Paper.p df

[23] D. A. Bowman and L. F. Hodges, "An evaluation of techniques for grabbing and manipulating remote objects in immersive virtual environments," in Proc. Symposium on Interactive 3D Graphics, 1997.

[24] M. Daily , M. Howard, J. Jerald , C. Lee, K. Martin, D. Mcinnes, P. Tinker, and R. C. Smith, "Distributed design review in virtual environments," in Proc. Third International Conference on Collaborative Virtual Environments, 2000, pp. 57-63.

[25] D. A. Bowman, "Interaction techniques for immersive virtual environments: design, evaluation, and application," Journal of Visual Languages and Computing, vol. 10, pp. 37-53, 1998.

[26] N. Ouramdane, S. Otmane, and M. Mallem, "3D interactions in virtual reality- state of art," Technique and computer Science Journal, vol. 28 , no. 8, pp. 1017-1049, 2009.

[27] A. Kunz and K. Wegener. Towards natural user interfaces in VR/AR for design and manufacturing. [Online]. Available: http://e-collection.library.ethz.ch/eserv/eth:6549/eth-6549-01.pdf

[28] M. R. Mine, "Virtual environment interaction techniques," Technical Report, University of North Carolina at Chapel Hill Chapel Hill, NC, USA, 1995.

[29] A. Reyes-Lecuona and A. Diaz-Estrella, "New interaction paradigms in virtual environments," in Proc. IEEE Mediterranean Electrotechnical Conference, 2006, pp. 449-452.

[30] F. A. Sanz and C. Andujar, "A survey of 3D object selection techniques for virtual environments," Computers and Graphics Journal, vol. 37 , no. 3, pp. 121-136, 2013.

[31] J. Jung, H. Park, D. Hwang, M. Son, D. Beck, J. Park, and W. Park, "A review on interaction techniques in virtual environments," in Proc. 2014 International Conference on Industrial Engineering and Operations Management, 2014, pp. 1582-1590.

[32] C. Weidig, D. R. Mestre, J. H. Israel, F. Noel, V. Perrot, and J. C. Aurich. Classification of VR interaction techniques, based on user intention. [Online]. Available: http://diglib.eg.org/bitstream/handle/10.2312/eurovr.20141339.053-0 58/053-058.pdf?sequence $=1$

[33] D. A. Bowman, E. Kruijff, L. Laviola, and I. Poupyrev, 3D User Interfaces: Theory and Practice, Addison-Wesley professional, CA, USA, 2004, ch. 5-8, pp. 139-285.

[34] D. J. Sturman, D. Zeltzer, and S. Pieper, "Hands-on interaction with virtual environments," in Proc. $2^{\text {nd }}$ Annual ACM SIGGRAPH Symposium on User Interface Software and Technology, 1989, pp. 19-24.

[35] R. A. Bolt, "Put-that-there: voice and gesture at the graphics interface," in Proc. 7th Annual Conference on Computer Graphics and Interactive Techniques, 1980, pp. 262-270.

[36] F. Steinicke, T. Ropinski, and K. Hinrichs, "Object selection in virtual environments with an improved virtual pointer metaphor," in Proc. International Conference on Computer Vision and Graphics, 2004, pp. 320-326.

[37] A. Olwal and S. Feiner, "The flexible pointer: An interaction technique for augmented and virtual reality," in Proc. ACM Symposium on User Interface Software and Technology, 2003, pp. 81-82.

[38] I. Poupyrev, S. Weghorst, M. Billinghurst, and T. Ichikawa, "The go-go interaction technique non-linear mapping for direct manipulation in VR," in Proc. 9th Annual ACM Symposium on User Interface Software and Technology, 1996, pp. 79-80.

[39] D. A. Bowman, "Interaction techniques for common tasks in immersive virtual environments: Design, evaluation, and application," Ph.D. dissertation, Georgia Institute of Technology, GA, USA, 1999.

[40] P. Boudoin, "Adaptive 3D interaction, an approach based on multi-sensor data processing methods," Ph.D. dissertation, Computer Science, Evry Val d'Essonne University, France, 2010.

[41] D. A. Bowman, J. Chen , C. A. Wingrave, J. Lucas, A. Ray, F. Nicholas, Q. Li, Y. Haciahmetoglu, J. S. Kim, S. Kim, R. Boehringer, and $\mathrm{T}$. Ni, "New directions in 3D user interfaces," International Journal of Virtual Reality, vol. 5, no. 2, pp. 3-14, 2006.

[42] Y. Dennemont, "Assistance to 3D interaction in virtual reality by semantic reasoning and context awareness," Ph.D. dissertation, Computer Science Department, Evry Val d'Essonne University, France, 2013.

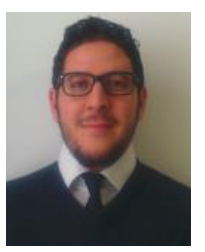

Mohammed Bougaa was born in 1988 in Algeria. He is a PhD student at EISTI, France; and is living at Paris. He got graduated as a computer sciences engineer in 2011 from the Higher National School of Computer Sciences, Algiers, Algeria, and received his master degree in virtual reality and smart systems from the Evry Val d'Essonne University in France, in 2013.

He held some positions such as a consultant at Microsoft Algeria and the IT manager at a cars distribution company before pursuing his studies in Virtual Reality Researches at France, where he did a six-months internship at Laval working on automatic generation of realistic human avatar with Professor Simon Richir at LAMPA Lab. He is interested in new fields of application of virtual reality, especially for education and systems engineering.

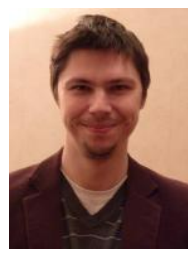

Stefan Bornhofen was born in 1972. He studied mathematics and computer science at the University of Mainz (Germany), and received a $\mathrm{PhD}$ degree in computer science from the University Paris-Sud, Orsay France in 2008. He fills a teaching and research position at the EISTI engineering school in Cergy near Paris, and is the head of a master's program specializing in computer graphics, computer vision and human-computer interaction. Stefan has strong interests in virtual worlds and immersive experiences. Current his research interests include virtual ecosystems, computer art and immersive CAO.

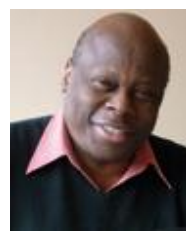

Hubert Kadima received his master degree in computer science and his $\mathrm{PhD}$ degree in automatics both from University of Paris VII in France. He has worked as a senior researcher and corporate level technical advisor for more than 30 years in a number of industrial research laboratories (ALCATEL, Jeumont Schneider, Bull ...). He has successfully coordinated several research projects funded by various funding European and French agencies. He has also a significant teaching university experience in computing science. Currently, he is the research director and the head of computing science EISTI-LARIS Laboratory at Cergy Pontoise in France. $\mathrm{He}$ has presented many publications in international and national conferences and has authored five books. His current research areas include distributed systems, cloud computing, semantic web and collaborative engineering systems.

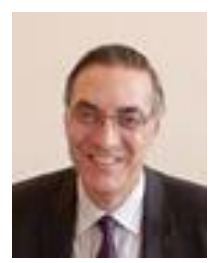

Alain Riviere is a full professor at the Institut Supérieur de Mécanique de Paris, Supméca. He received the PhD title from the Ecole Central de Paris, in 1993. Now he is the general director of Supméca. Prof. Alain Riviere is author or co-author of two scientific books and more than sixty scientific papers related to CAD-CAM designing, with a particular focus on geometrical product specification. He is a member of the French Association of Mechanics (AFM). He was the director of LISMMA Lab for five years, from 2005 to 2010 . 